Rechtsmedizin 2020 · 30:430-437

https://doi.org/10.1007/s00194-020-00421-0

Online publiziert: 9 . September 2020

(c) Der/die Autor(en) 2020

N. Kern · F. Holz · M. A. Verhoff · M. Parzeller

Institut für Rechtsmedizin, Universitätsklinikum, Goethe-Universität Frankfurt, Frankfurt am Main Deutschland

\title{
Befragung von \\ Medizinstudierenden zu der ärztlichen Aufklärung und zu den ärztlichen Informationspflichten sowie zur medizinrechtlichen Ausbildung im Studium
}

in $\$ 115$ S. 2 Nr. 5 ÄApprO-E auf Kenntnisse wichtiger arzneimittelrechtlicher Vorschriften als Inhalt des Vierten Abschnitts der Ärztlichen Prüfung hingewiesen. In $₫ 159$ Abs. 1 S. 2 ÄApprO-E (Kenntnisprüfung für ausländische Ärzte/Ärztinnen) finden sich Vorgaben zur Stellung von Prüfungsfragen zu Rechtsfragen der ärztlichen Berufsausübung. Erst in den Anlagen zur ÄApprO-E wird auf rechtliche Aspekte abgestellt: Recht der Medizin in Anlage 3 (übergeordnete Kompetenzen, die Teil der bis zum Ersten, zum Zweiten und zum Dritten Abschnitt der Ärztlichen Prüfung $\mathrm{zu}$ erbringenden Leistungsnachweise sind), rechtliche Aspekte im Kontext der Transplantationsmedizin und des Schwangerschaftsabbruchs; Rechtsfragen der ärztlichen Berufsausübung in Anlage 14 (klinischer Prüfungsstoff für den Ersten, den Zweiten und den Dritten Abschnitt der Ärztlichen Prüfung) sowie Recht der Medizin in Anlage 15 (übergeordneter, kompetenzbezogener Prüfungsstoff für den Ersten, den Zweiten und den Dritten Abschnitt der Ärztlichen Prüfung). Um den rechtlichen Aspekten im Medizinstudium besser Rechnung zu tragen, wurde aus dem Institut für Rechtsmedizin in Frankfurt ein Entwurf erarbeitet (• Abb. 1), der vonseiten des Berufsverbandes Deutscher Rechtsmediziner e. V. und der Deutschen Gesellschaft für Rechtsmedizin für ei- ne gemeinsame Stellungnahme an das Bundesgesundheitsministerium genutzt wurde.

Gemäß $₫ 3$ Abs. 4 ÄApprO dient der letzte Abschnitt des Humanmedizinstudiums, das praktische Jahr (PJ), der Aneignung und Festigung praktischer Fertigkeiten, für die während des vorhergehenden Studiums die theoretischen Grundlagen gelegt wurden [1]. Um in dieser Zeit optimal auf den ärztlichen Berufsalltag vorbereitet zu werden, sollten Studierende so viel wie möglich eigenständig, jedoch unter ärztlicher Anleitung oder Aufsicht, ausführen [1]. Nicht nur für die ärztliche Berufstätigkeit ist der Umgang mit Medizinrecht von großer Bedeutung, sondern schon im Studium werden angehende Ärzte/Ärztinnen mit juristischen Fragen konfrontiert. Sowohl während diverser Famulaturen als auch im PJ sind Studierenden die entsprechenden rechtlichen Grundlagen häufig unklar. Hierbei kann es gerade im hektischen Klinikalltag und bei herrschendem Personalmangel zu Verwirrungen bei der Frage kommen: „Was dürfen PJ-Studierende im Krankenhaus eigentlich für Aufgaben übernehmen?“ [3]. Dies kann dazu führen, dass die Delegation von Aufgaben an Studierende unklar bleibt und es zu Kompetenzüberschreitungen (Übernahmeverschulden) kommt. Die Vergangenheit lehrt, dass Unsicherheiten in Bezug 
Die ärztliche Tätigkeit wird von einer Vielzahl normativer Vorgaben geregelt. Dem Arzt-, Medizinund Patientenrecht kommt eine wichtige Bedeutung zu, was u. a. durch die Änderungen im BGB durch das Patientenrechtegesetz unterstrichen wurde. Nicht nur historische und ethische Grundlagen, sondern gerade rechtliche Aspekte sind für ärztliches Handeln von entscheidender Bedeutung. Die Vermittlung von Rechtskenntnissen insbesondere im Zusammenhang mit Arztund Patientenrechten ist für die Ausbildung zu einer verantwortungsvollen ärztlichen Tätigkeit unerlässlich.

Der aktuelle Entwurf weist unmittelbar in der ÄApprO-E bisher bezüglich des Arzt-, Medizin- und Patientenrechts für das zukünftige Studium lediglich in § 1 Abs. $2 \mathrm{~S}$. $3 \mathrm{Nr}$. 4 ÄApprO-E auf relevante Kenntnisse, Fähigkeiten und Fertigkeiten hinsichtlich der datenschutzrechtlichen Grundlagen und in $\S 115$ S. 2 Nr. 5 ÄApprO-E auf Kenntnisse wichtiger arzneimittelrechtlicher Vorschriften als Inhalt des Vierten Abschnitts der Ärztlichen Prüfung hin.

Des Weiteren findet sich in $\S 159$ Abs. 1 S. 2 ÄApprO-E der Hinweis auf die Stellung von Prüfungsfragen zu Rechtsfragen der ärztlichen Berufsausübung in der Kenntnisprüfung für ausländische Ärztlinnen.

Erst in den Anlagen zur ÄApprO-E wird auf rechtliche Aspekte abgestellt: Anlage 3 (Recht der Medizin), Anlage 14 (rechtliche Voraussetzungen bei der Entnahme und Übertragung von Organen und Geweben; rechtliche Aspekte des Schwangerschaftsabbruchs; Rechtsfragen der ärztlichen Berufsausübung), Anlage 15 (Recht der Medizin)

Es würde sich daher folgende kurze Einfügung in $\S 1$ Abs. 2 S. 2 Nr. 8 ÄApprO-E als Ziel der Ausbildung empfehlen:

\begin{tabular}{|l|l|}
\hline $\begin{array}{l}\text { Arbeitsentwurf der BMG: } \\
\text { 8. die theoretischen, historischen und } \\
\text { ethischen Grundlagen ärztlichen Handelns }\end{array}$ & $\begin{array}{c}\text { Vorschlag der/des DGRM/BDRM: } \\
\text { 8. die theoretischen, historischen, rechtlichen } \\
\text { und ethischen Grundlagen ärztlichen Handelns }\end{array}$ \\
\hline
\end{tabular}

Des Weiteren sollte $§ 115$ S. 2 ÄApprO-E (Inhalt des Vierten Abschnitts der Ärztlichen Prüfung) um Nr. 16 ergänzt werden:

Vorschläge:

16. die Grundlagen des Arzt-, Medizin- und Patientenrechts beherrscht.

oder

16. die Grundlagen des Rechts für das ärztliche Handeln beherrscht.

Der Eindruck, dass bei den Zielen der Ausbildung lediglich auf das Datenschutzrecht abgestellt wird, könnte durch die Anderung in § 1 Abs. 2 S. 2 Nr. 8 AApprO-E durch die Einbeziehung des Rechts als Ausbildungsziel vermieden werden. Mit der Ergänzung in § 115 S. 2 Nr. 16 ÄApprO-E beim Vierten Abschnitt der Ärztlichen Prüfung würde sich der Stellenwert des Rechts unmittelbar in der ÄApprO nicht nur auf die Kenntnisprüfung bei ausländischen Ärztlinnen beschränken,

sondern das Recht wäre als wichtiger Prüfungspunkt unmittelbar in der ÄApprO (in Ergänzung zu

den Anlagen) verankert. Der mögliche Eindruck von überwiegend arzneimittelrechtlich erforderlichen Kenntnissen der Studierenden in dieser Prüfung könnte somit vermieden werden.

Abb. $1 \Delta$ Vorbereitende Stellungnahme zum Arbeitsentwurf der Approbationsordnung für Ärzte und Ärztinnen (ÄApprO) des Bundesministeriums für Gesundheit von Parzeller vom 23.01.2020 für die Deutsche Gesellschaft für Rechtsmedizin und den Berufsverband Deutscher Rechtsmediziner e.V. zur Einreichung beim Bundesgesundheitsministerium (BMG)

auf rechtliche Rahmenbedingungen und delegierbare Tätigkeiten in Einzelfällen fatale Folgen haben können $[4,5]$.

Obwohl Medizinrecht im ärztlichen Berufsalltag einen hohen Stellenwert einnimmt und schon in den praktischen Abschnitten des Medizinstudiums einen wichtigen Teil der Patientensicherheit darstellt, werden rechtliche Aspekte nur in geringem Umfang und vorwiegend von der Rechtsmedizin behandelt (zum Lehrangebot der Rechtsmedizin in Frankfurt: [6]), und „Pflichtveranstaltungen werden zumeist fachspezifisch durchgeführt" [7]. Jedoch stellt die Rechtsmedizin ein vergleichsweise kleines Fach während des Medizinstudiums mit einem kleinen Lehrkontingent dafür vorgesehene Zeit im Curriculum ist stark begrenzt. Noch im Jahr 2017 war nur „in 66,7 \% der universitären Institute für Rechtsmedizin die Ableistung eines PJ-Tertials möglich“ [9], und teilweise führten Änderungen der Approbationsordnung in der Vergangenheit an manchen Universitäten sogar zu einer „drastischen Reduktion der rechtsmedizinischen Unterrichtsinhalte" [10]. Obwohl die medizinische Ausbildungsforschung in den vergangenen Jahren an
Bedeutung zugenommen hat [11], und während andere Kernkompetenzen, wie beispielsweise die Medizinethik, immer stärker in das Studium und den Klinikalltag integriert werden [12], fehlen solche Strukturen für das Medizinrecht noch weitestgehend. Einer Studie zufolge sieht "die ganz große Mehrheit [von Studierenden im Praktischen Jahr] die Vermittlung von Rechtskenntnissen als sehr wichtig bzw. als außerordentlich wichtig an" und kommt zu dem Schluss, dass eine „Bereitstellung eines Lehrbeauftragten für Medizinrecht" als sinnvoll $\mathrm{zu}$ erachten ist [13].

Die Befragung in dieser Studie orientierte sich schwerpunktmäßig an derärztlichen Aufklärung. Es handelt sich dabei um eine elementare und wichtige Aufgabe in der späteren ärztlichen Berufsausübung, insbesondere weil das OLG Karlsruhe sogar eine alleinige Aufklärung einer Patientin durch eine Medizinstudierende im PJ über eine Herzkatheteruntersuchung für zulässig erachtet hatte. Seine Entscheidung hat das OLG Karlsruhe u. a. damit begründet, dass „die Aufklärung durch einen solchen Studenten [...] der ärztlichen Aufklärung gleichstehen kann" [16]. Aufgrund der zwischenzeitlich erfolgten Vorgaben zur ärztlichen Aufklärung im BGB ( $\$ 630$ e BGB) durch das PatRG ist diese Entscheidung umstritten und mit erheblichen Haftungsrisiken verbunden (ausführlich: [14, 20]). Um den Fragebogen nicht zu überfrachten, wurden andere relevante Aspekte des Medizinrechts, wie z.B. die ärztliche Schweigepflicht, die ärztlichen Dokumentationspflichten oder der Umgang mit möglichen ärztlichen Behandlungsfehlern nicht abgefragt.

\section{Material und Methoden}

Im Zeitraum zwischen Sommersemester 2017 und Sommersemester 2019 wurden in verschiedenen Jahrgängen des Regelstudienganges Humanmedizin an der Goethe-Universität Frankfurt am Main zu Beginn des rechtsmediziniSemester standardisierte Fragebogen (- Abb. 2) verteilt. Der Unterschied des klinischen Semesters ergibt sich aus dem Umstand, dass die Medizinstudierenden schen Kurses im 4. bzw. 5. klinischen 
Rechtsmedizin 2020 · 30:430-437 https://doi.org/10.1007/s00194-020-00421-0

(c) Der/die Autor(en) 2020

\section{N. Kern · F. Holz · M. A. Verhoff · M. Parzeller}

\section{Befragung von Medizinstudierenden zu der ärztlichen Aufklärung und zu den ärztlichen Informationspflichten sowie zur medizinrechtlichen Ausbildung im Studium}

\section{Zusammenfassung}

Hintergrund. Mit dem Masterplan 2020 und den an mehreren Universitäten eingeführten Modellstudiengängen befindet sich das Medizinstudium aktuell im Umbruch. Sowohl im Regel- als auch im Modellstudiengang werden medizinrechtliche Aspekte überwiegend im Rahmen rechtsmedizinischer Ausbildungsabschnitte unterrichtet. Allerdings werden Studierende bereits während Famulaturen oder im praktischen Jahr mit juristischen Fragen konfrontiert.

Ziel der Studie war es herauszufinden, ob und in welchem Umfang Studierende der Humanmedizin insbesondere zur ärztlichen Aufklärung und zu den ärztlichen Informationspflichten bis zum Beginn des 4. bzw. 5. klinischen Semesters auf medizinrechtliche Aspekte vorbereitet wurden, und ob Verbesserungen bei der medizinrechtlichen Lehre gewünscht werden. Material und Methoden. Zwischen den Sommersemestern 2017 und 2019 wurde zu Beginn des Kurses für Rechtsmedizin eine quantitative, standardisierte Umfrage mit insgesamt 373 Studierenden durchgeführt. Ergebnisse. Wenngleich 98,8\% der Studierenden angaben, Aufklärungsgespräche bereits (mehrfach) praktisch ausgeübt zu haben, bestanden deutliche Defizite in Bezug auf die juristischen Anforderungen an das ärztliche Aufklärungsgespräch und dessen Delegation. So gaben lediglich 5,1\% der Studierenden an, die rechtlichen Grundlagen der ärztlichen Aufklärung sowie die entsprechende Norm aus dem Zivilrecht zu kennen. Über $80 \%$ der Befragten fühlten sich unzureichend auf die rechtlichen Aspekte des praktischen Jahres vorbereitet. Über $90 \%$ der Studierenden wünschten sich eine bessere medizinrechtliche Ausbildung.

Diskussion. Eine fächerübergreifende Etablierung sowie eine über das gesamte Studium verteilte Lehre von Medizinrecht könnte die Vorbereitung auf das praktische Jahr verbessern und das Verständnis für die rechtlichen Anforderungen an die ärztliche Berufstätigkeit fördern. Nach dem derzeitigen Stand der Umsetzung des Masterplans 2020 soll das Medizinrecht in der Learning Opportunities, Objectives and Outcomes Platform (LOOOP) als verbindlicher Ausbildungs- und Lehrinhalt etabliert werden.

Schlüsselwörter

Medizinrecht · Medizinstudium · Praktisches Jahr · Masterplan 2020 · Delegation

\section{Survey of medical students on medical clarification and medical information obligations as well as on the improvement of general medicolegal education}

\section{Abstract}

Background. Medical studies are currently undergoing a radical change through the Master Plan 2020 and the introduction of model courses at several universities. Within the standard and model study programs aspects of medical law are mainly taught during forensic medicine training sections. Students are already confronted with legal questions during their clinical traineeships or practical year.

The objective of the study was to find out whether and to what extent medical students were prepared for the medicolegal aspects by the beginning of the 4th or 5th clinical semester, particularly with specific respect to medical clarification and medical information obligations and whether improvements in medicolegal teaching are desired.
Material and methods. A quantitative standardized survey was conducted with a total of 373 students at the beginning of each forensic medicine course between the summer semesters 2017 and 2019. Results. Nearly all students (98.8\%) stated that they had already practically carried out explanatory conversations several times before the start of the forensic medicine course; however, there were significant deficits in theoretical knowledge of the medical explanatory conversation and its delegation. Only $5.1 \%$ of the students stated that they were familiar with the legal principles of medical clarification and the corresponding norm found in civil law. More than $80 \%$ of participants felt insufficiently prepared for the legal aspects of the practical year. More than
$90 \%$ of students wished for a better training in medical law.

Discussion. Establishment of the interdisciplinary teaching of medical law spread over the entire course of studies could improve students' preparation for the practical year and promote their understanding of the legal requirements for the medical profession. According to current developments in the implementation of the Master Plan 2020, medical law should be established in the learning opportunities, objectives and outcomes platform (LOOOP) as obligatory training and teaching content.

Keywords

Medical law · Medical studies - Year of practical training · Master Plan 2020 · Delegation zwischen zwei Kohorten (Chirurgie und innere Medizin) wählen können und dadurch die verschiedenen Fächer versetzt durchlaufen. Alle Befragten hatten vor dem Zeitpunkt der Umfrage weder die rechtsmedizinische Begleitvorlesung noch den rechtsmedizinischen Kurs mit seinem theoretischen und praktischen Teil besucht. Vereinzelte Studierende könnten jedoch einige Jahre vor der Befragung an dem vorklinischen Wahlfach „Interdisziplinäres medizinrechtliches Seminar" mit einer Teilnehmeranzahl von maximal 10 Medizinstudierenden teilgenommen haben.

$\mathrm{Zu}$ Beginn des Fragebogens wurden der Hintergrund der Umfrage skizziert und die anonymisierte Auswertung der Daten zugesichert.

Der Erhebungsfragebogen, bestehend aus 8 Fragen, wurde in 2 Teilbereiche untergliedert. Der erste Teilbereich (Fragen 1-6) befasste sich mit unterschiedlichen Fragestellungen zur ärztlichen Aufklärung und zu den ärztlichen Informationspflichten. Dabei wurde analysiert, 


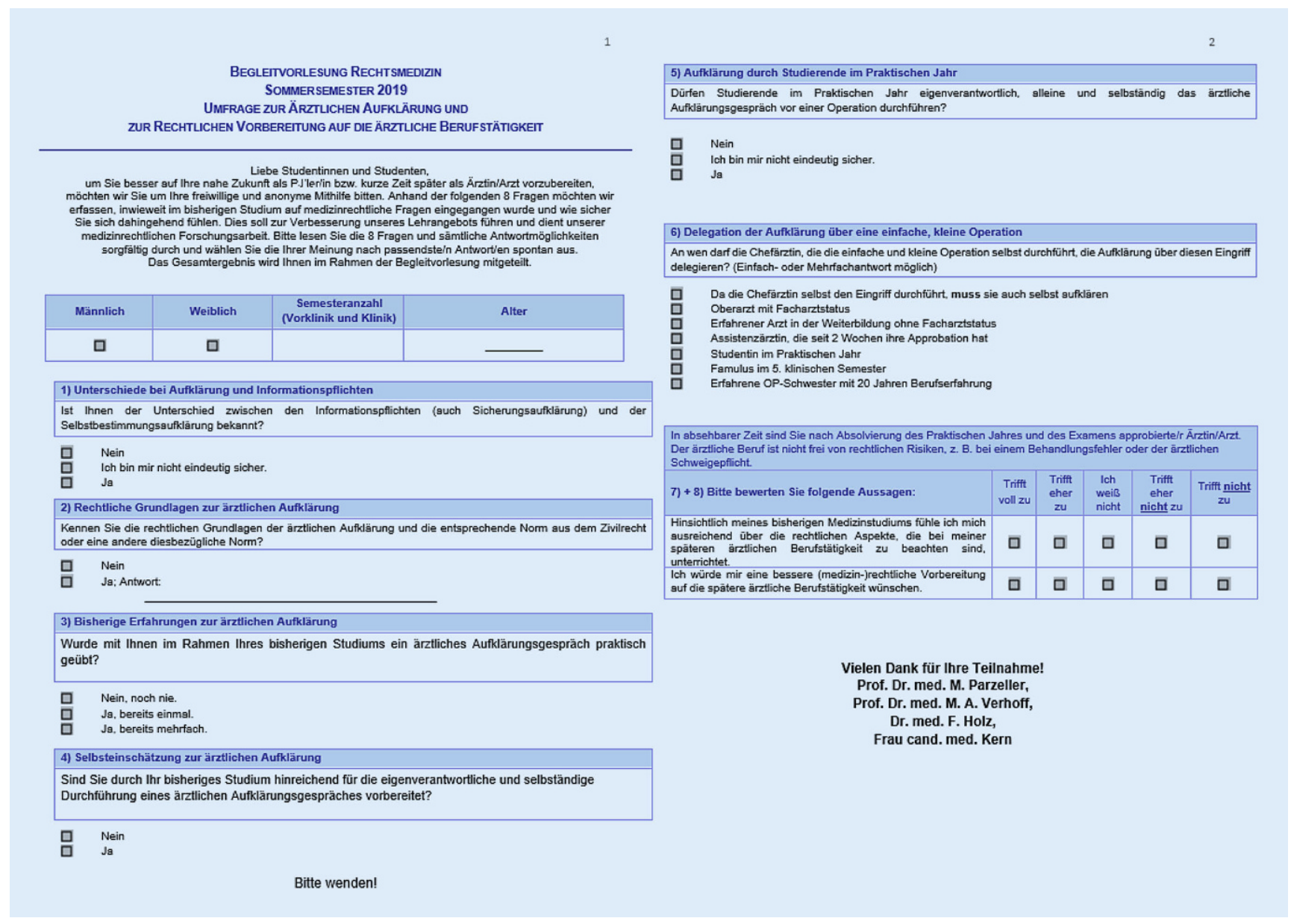

Abb. 2 \ Standardisierter Fragebogen „Begleitvorlesung Rechtsmedizin - Umfrage zur ärztlichen Aufklärung und zur rechtlichen Vorbereitung auf die ärztliche Berufstätigkeit" als Version Sommersemester 2019

welche rechtlichen Grundlagen den Studierenden bekannt sind, und welche eigenen praktischen Erfahrungen sie bereits in ihrem bisherigen Studium hinsichtlich des ärztlichen Aufklärungsgesprächs erfahren haben. Anhand von Beispielfragen zur „Aufklärung der Studierenden im PJ" und zur Delegation der Aufklärung an verschiedene Personengruppen im medizinischen Bereich wurde diesbezügliches Wissen eruiert.

Der zweite Teilbereich (Fragen 7 und 8) warf, unter Hinweis auf die rechtlichen Risiken beispielsweise bei Behandlungsfehlern und der ärztlichen Schweigepflicht, die Frage auf, ob sich die Studierenden medizinrechtlich hinreichend für ihre spätere ärztliche Berufstätigkeit durch das Studium unterrichtet fühlen. Zudem erfolgte eine Abfrage, ob hinsichtlich der medizinrechtlichen Vorbereitung für die spätere ärztliche Berufstätigkeit Verbesserungen gewünscht sind.

\section{Ergebnisse}

Insgesamt füllten 373 Studierende (250 weiblich, 121 männlich, 2 ohne Angabe) im Alter zwischen 21 und 55 Jahren (Mittelwert: 23 Jahre, Median: 23 Jahre) den Fragebogen vollständig aus.

Die Umfrage ergab, dass deutliche Defizite in Bezug auf die theoretischen Kenntnisse des ärztlichen Aufklärungsgesprächs und dessen Delegation bestanden. Trotz vorangegangener praktischer Übungen zu Aufklärungsgesprächen und absolvierter Famulaturen gaben knapp zwei Drittel (62,2\%) der Studierenden an, den Unterschied zwischen den Informationspflichten (Sicherungsaufklärung) und der Selbstbestimmungsaufklärung nicht zu kennen; 32,4\% seien sich nicht eindeutig sicher. Lediglich $5,4 \%$ antworteten, ihnen sei der Unterschied bekannt.
Die Frage, ob den Studierenden die rechtlichen Grundlagen der ärztlichen Aufklärung und die entsprechende Norm aus dem Zivilrecht oder eine andere diesbezügliche Norm bekannt seien, beantworteten $94,9 \%$ mit ,nein“. Von den 5, $1 \%$ der Studierenden, die diese Frage mit „ja “ beantworteten, nutzten lediglich 8 Studierende die Freitextangabe. Hierbei wurde nur in einer Antwort ein Paragraf ( $\$ 630$ BGB) genannt; die restlichen Antworten waren nicht aussagekräftig.

Im Gegensatz zu den theoretischen Grundlagen gaben nahezu alle Studierenden $(98,8 \%)$ an, das ärztliche Aufklärungsgespräch im Laufe des Studiums mindestens einmal, in den meisten Fällen bereits mehrfach praktisch geübt zu haben. Zwei Drittel der Studierenden (66,0\%) führten aus, durch ihr bisheriges Studium „hinreichend für die eigenverantwortliche und selbstständige Durch- 


\section{Originalien}

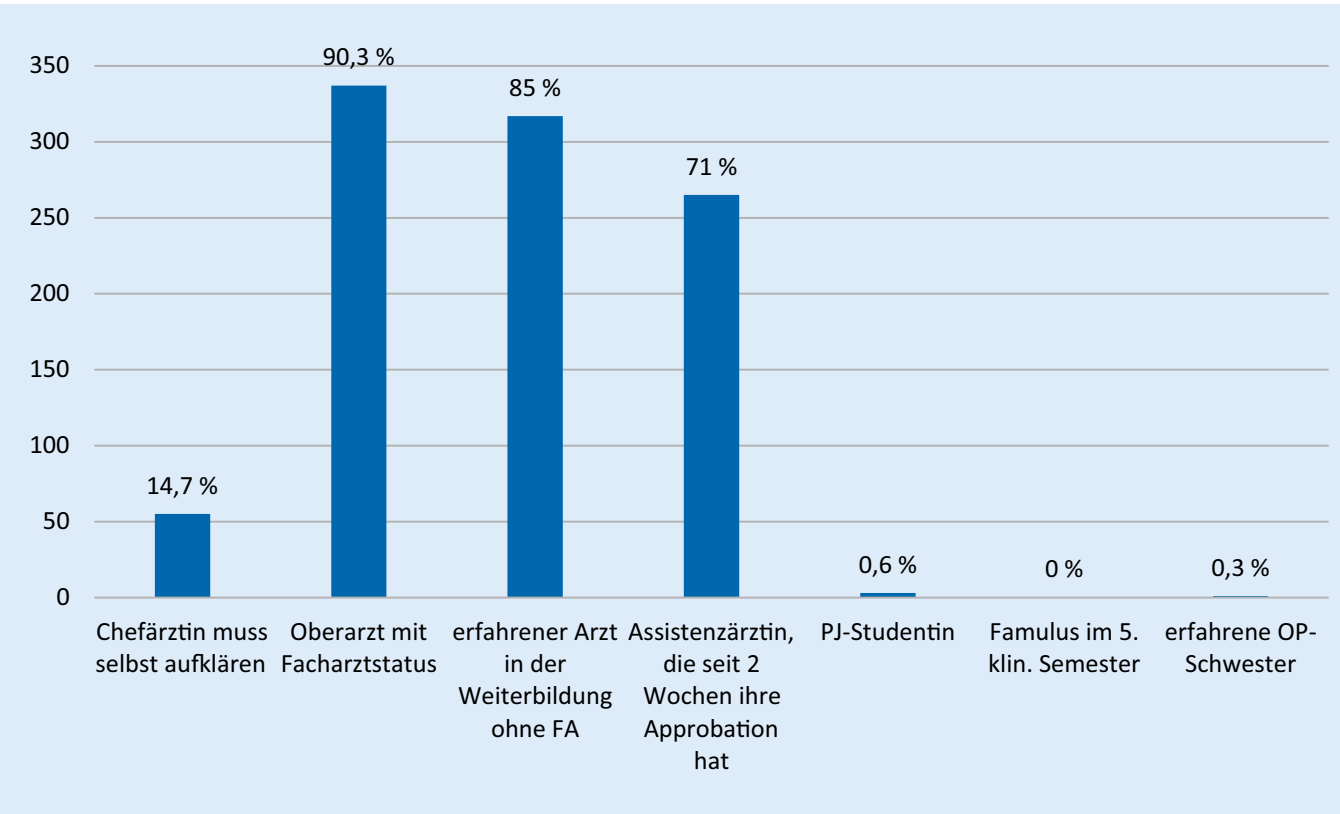

250

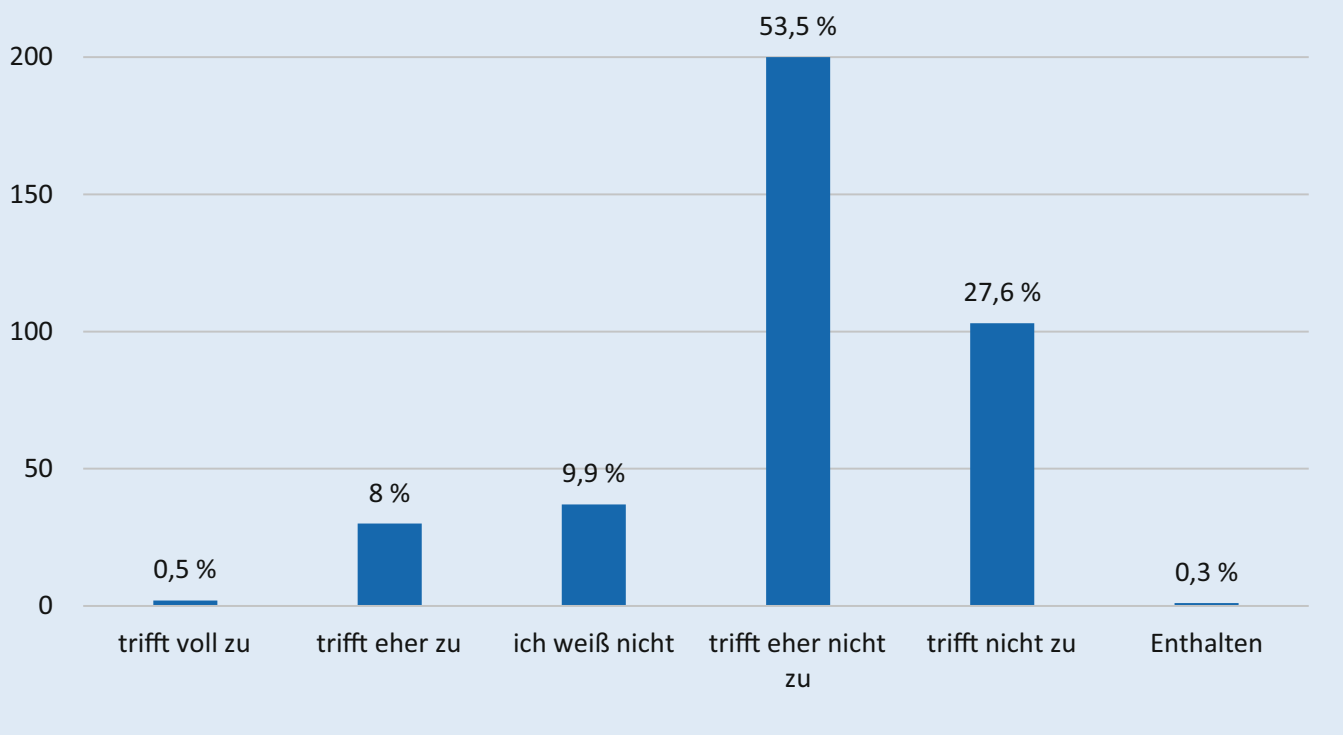

Abb. $3<$ Prozentuale Verteilung der Antworten auf die Frage: „An wen darf die Chefärztin, die die einfache und kleine Operation selbst durchführt, die Aufklärung über diesen Eingriff delegieren? (Einfach-oder Mehrfachantwort möglich)"
Abb. $4 \triangleleft$ Prozentuale Verteilung: „Hinsichtlich meines bisherigen Medizinstudiums fühle ich mich ausreichend über die rechtlichen Aspekte, die bei meiner späteren ärztlichen Berufstätigkeit zu beachten sind, unterrichtet" führung eines ärztlichen Aufklärungsgespräches" vorbereitet zu sein.

Im Hinblick auf Medizinrecht im PJ gaben 93,8\% der Befragten an, dass sie als PJ-Student nicht eigenverantwortlich und allein Aufklärungsgespräche vor operativen Eingriffen durchführen dürfen. Im Weiteren wurde ein Fallbeispiel präsentiert, bei dem eine Chefärztin eine einfache und kleinere Operation durchführen, jedoch das Aufklärungsgespräch vor dem Eingriff delegieren möchte. Während 14,7\% der Befragten angaben, die Aufklärung sei nicht delegierbar, gaben 3 Studierende $(0,8 \%)$ an, dass ein PJ-Student die Aufklärung eigenständig und allein durchführen könne. Unter den vorgegebenen Mehrfachantworten fanden sich im Weiteren „Oberarzt mit Facharztstatus“, „erfahrener Arzt in der Weiterbildung, ohne Facharztstatus“, „Assistenzärztin, die seit 2 Wochen ihre Approbation hat", „Famulus im 5. klinischen Semester" sowie „erfahrene OP-Schwester mit 20 Jahren Berufserfahrung“. Die prozentuale Verteilung der durch die Studierenden gegebenen Antworten zeigt • Abb. 3 .

Bei den Fragen nach der subjektiven Einschätzung hinsichtlich der medizinrechtlichen Ausbildung im bisherigen Studium fühlten sich $81,2 \%$ der Studierenden „nicht“ oder „eher nicht“ ausreichend unterrichtet (- Abb. 4). Mit 92\% gab die überwiegende Mehrheit an, sich eine bessere (medizin-)rechtliche Aus- 


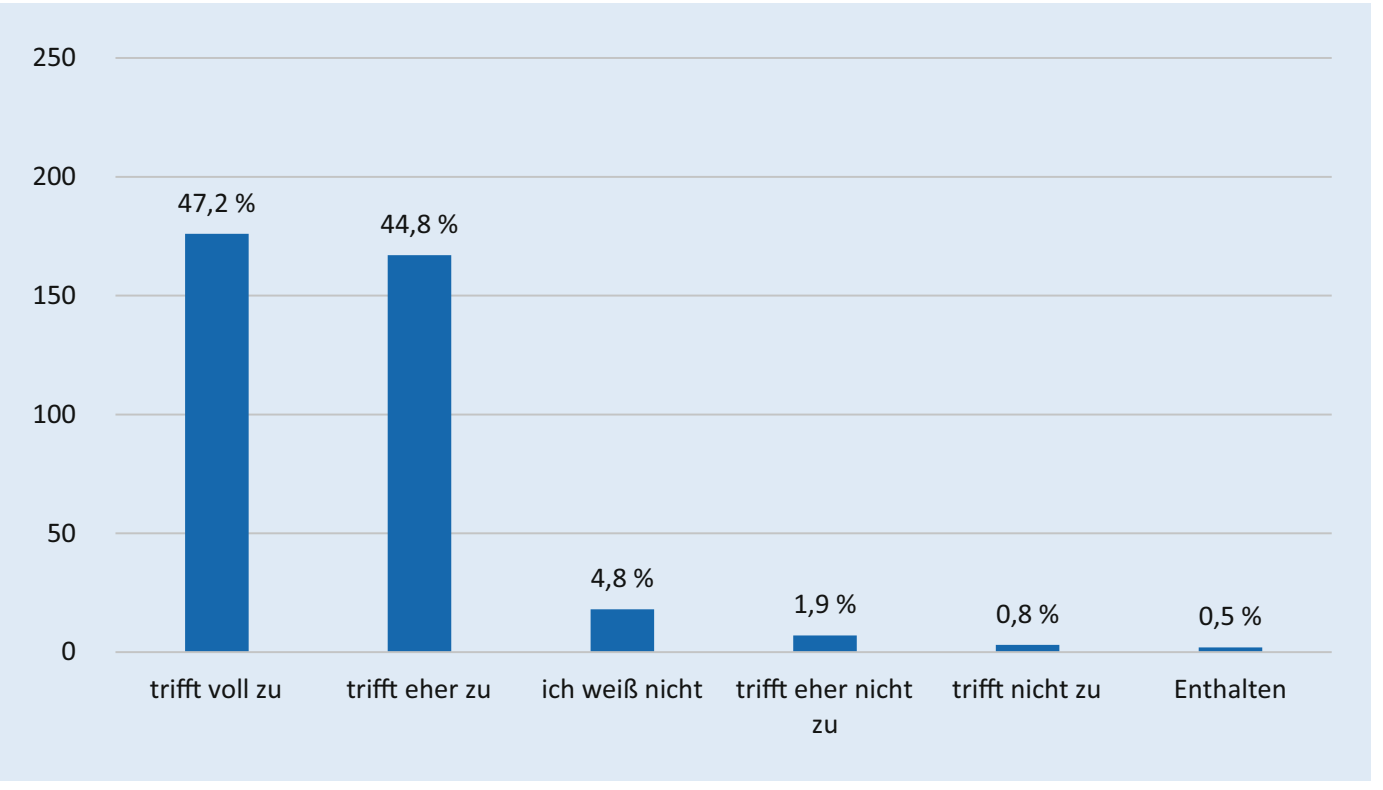

Abb. $5 \triangleleft$ Prozentuale Verteilung: „Ich würde mir eine bessere (medizin-)rechtliche Vorbereitung auf die spätere ärztliche Berufstätigkeit wünschen" bildung als Vorbereitung auf den späteren ärztlichen Berufsalltag zu wünschen (• Abb. 5).

\section{Diskussion}

Studierende im PJ haben es oft nicht leicht: Einerseits „sollen die Studierenden die während des vorhergehenden Studiums erworbenen ärztlichen Kenntnisse, Fähigkeiten und Fertigkeiten vertiefen und erweitern" [1]; andererseits muss der rechtlich abgesicherte Patientenschutz im Vordergrund stehen, was die Möglichkeit zum Üben einschränkt und die Studierenden verunsichert. So behandelten bereits mehrere Publikationen die Delegation der ärztlichen Aufklärung bzw. anderer ärztlicher Tätigkeiten an (PJ-)Studierende mitunter ablehnend [14, 15, 17-20]. In Einzelfallentscheidungen wurde dieses Problem auch in der Rechtsprechung abgehandelt [16].

Um einen ersten Überblick über den Wissenstand von Studierenden im medizinrechtlichen Bereich $\mathrm{zu}$ erhalten, wurde die Fragebogenerhebung auf die wichtigen Punkte der ärztlichen Aufklärung und der Informationspflichten fokussiert. Es besteht nach den Erfahrungen der Studienautoren jedoch ein deutliches Indiz dafür, dass auch in anderen Bereichen des Medizinrechts bei den Studierenden erhebliche Wissenslücken vorliegen dürften. So führt selbst der
Gesetzgeber in der Gesetzesbegründung zum PatRG aus: „Welche Rechte Patientinnen und Patienten haben, wissen daher oftmals weder sie selbst noch die Behandelnden" [21].

Diese Studie zu Beginn des Rechtsmedizinkurses zeigt, dass viele Befragte Defizite in Bezug auf die medizinrechtlichen Grundlagen für den anstehenden Berufsalltag aufwiesen. Ein Drittel der Befragten gab an, durch ihr bisheriges Studium nicht ,hinreichend für die eigenverantwortliche und selbstständige Durchführung eines ärztlichen Aufklärungsgespräches" vorbereitet zu sein. Hinsichtlich der medizinrechtlichen Ausbildung fühlten sich $81,2 \%$ der Studierenden im bisherigen Studium "nicht" oder "eher nicht" ausreichend unterrichtet. Sowohl die Arbeit von Neuser et al. [13] als auch diese Studie belegen, dass Studierende sowohl im PJ als auch bereits in der klinischen Phase des Medizinstudiums die Notwendigkeit einer medizinrechtlichen Wissensvermittlung im Studium aufzeigen und sich eine bessere (medizin-)rechtliche Vorbereitung auf die spätere ärztliche Berufstätigkeit wünschen.

Da Medizinstudierende bereits während ihres Studiums mit medizinrechtlichen Fragen konfrontiert werden, sind solche Defizite im Studium nicht hinnehmbar. Bereits bei der Absolvierung von Famulaturen und Blockpraktika müssten theoretische Grundkenntnisse, auch über medizinrechtliche Aspekte, vorhanden sein und teils angewandt werden [22, 23]. Medizinrecht findet jedoch häufig keinen eigenen Platz im Curriculum der medizinischen Universitäten. Nach den Empfehlungen der Expertenkommission zum Masterplan 2020 wäre eine Einbindung des Medizinrechts über Brückenstudienfächer in Form optionaler Lehrveranstaltungen oder Wahlpflichtfächer möglich, und das Recht wird in den Vorschlägen für einen neuen Leistungskatalog aufgeführt [24]. Diesbezüglich kann angeführt werden, dass „die Rechtsmedizin als Medizin mit ihren ureigenen medizinisch-naturwissenschaftlichen Methoden auf die Rechtsordnung bezogen ist, während das Medizinrecht in seiner Eigenschaft als Recht seine Rechtsregeln auf die Medizin ausrichtet" [25]. Das Medizinrecht durchzieht alle medizinischen Fachrichtungen. Es ist essenzieller Bestandteil des ärztlichen Handelns und muss im Studium unabhängig von der Rechtsmedizin behandelt und von den Studierenden theoretisch wie praktisch beherrscht werden können. Zudem sollten die Grundlagen des Medizinrechts von allen Lehrenden berücksichtigt und idealerweise auf die tägliche Arbeit im Krankenhaus übertragen werden können [25].

Durch die Aufteilung in den klassischen und den seit über 20 Jahren beste- 
henden Modellstudiengang [26] genauso wie durch uneinheitliche Curricula [27] verschiedener Universitäten und den unterschiedlichen Einsatz neuer Lehr- und Lernmethoden wie E-Learning-Module [28] ist ein Vergleich der medizinrechtlichen Ausbildung erschwert. Vor allem aus dem Vereinigten Königreich kamen in der Vergangenheit Studien, die die Bedeutung von Medizinrecht im Medizinstudium aufzeigten und nach Konzepten für die Lehre medizinrechtlicher Inhalte sowie für die Bewertung von Studierenden in diesem Bereich suchten [29-31]. Dort konnte gezeigt werden, dass Studierende von einer medizinrechtlichen Ausbildung in den ersten Jahren des Studiums profitieren konnten [32]. Neuere Studien setzten auf das sog. angewandte Recht [33], bei dem die juristische Seite gleichwertig zu medizinischen Inhalten gelehrt wird.

Das "Safety Curriculum“ der Weltgesundheitsorganisation (WHO) ist darauf ausgerichtet, die Patientensicherheit durch eine strukturiertere Ausbildung im Medizinstudium zu erhöhen [34]. Die WHO empfiehlt die Einführung in das Medizinrecht bereits im ersten Jahr der medizinischen Ausbildung. Gemäß dem „Safety Curriculum“ dient es der Patientensicherheit, wenn rechtliche Grundlagen schon früh in der Ausbildung und durch häufige Wiederholungen gefestigt werden. Die vorliegende Umfrage zeigt, dass eine frühzeitige Etablierung von medizinrechtlichen Aspekten im Studium der Humanmedizin dem Wunsch von $92 \%$ der Befragten entsprechen würde, um dadurch eine bessere (medizin-)rechtliche Ausbildung als Vorbereitung auf den späteren ärztlichen Berufsalltag zu erhalten.

Die aktuellen Entwicklungen bei der Reform des Medizinstudiums als auch bei der Weiterentwicklung der Nationalen Kompetenzbasierten Lernzielkataloge Medizin (NKLM) und der Gegenstandskataloge (GK) des Instituts für medizinische und pharmazeutische Prüfungsfragen (IMPP) tragen dieser Verrechtlichung der ärztlichen Berufstätigkeit deutlich Rechnung und nehmen medizinrechtliche Themen in die Ausbildungsinhalte der jeweiligen Kataloge auf. Im Netzwerk „Learning Opportuni- ties, Objectives and Outcomes Platform" (LOOOP) ist bereits ein umfassendes eigenständiges Kapitel zum Medizinrecht, nebst einem Glossar, eingefügt worden, und über das Kapitel „Professionelles Handeln" werden zudem rechtliche Inhalte abgebildet. Der Medizinrechtskatalog wurde in LOOOP am 06.03.2020 freigeschaltet [35]. Auf Basis der Entwürfe zu rechtlichen Fragestellungen im Kapitel „Professionelles Handeln“, Ethik, Geschichte und Recht der Medizin erfolgte bereits eine Aufnahme dieser Prüfungsinhalte in den GK des IMPP [36]. So enthält die 5. aktualisierte Auflage des IMPP-GK2 des IMPP vom November 2019 ein Kap. 5 zur Kenntnis und zur Berücksichtigung medizinrechtlicher Aspekte, z.B. zu den „Voraussetzungen und [den] zivilrechtlichen Vorgaben für den Behandlungsvertrag, insbesondere hinsichtlich der Informationspflichten (auch Fehleroffenbarungspflicht), der Selbstbestimmungsaufklärung, der Einwilligung von (inklusive minderjähriger oder einwilligungsunfähiger) Patientinnen und Patienten, der Dokumentationspflichten, des Einsichtsrechtes von Patientinnen und Patienten und der Beweislastgrundsätze“ [36]. Um die Medizinstudierenden frühzeitig mit medizinrechtlichen Belangen vertraut $\mathrm{zu}$ machen, empfiehlt es sich, Grundlagen des Medizinrechts bereits in der Vorklinik über ein für alle Medizinstudierende obligatorisches vorklinisches Wahlfach etc. (s. bereits zur Lehre des Instituts für Rechtsmedizin in Frankfurt/Main [6]) $\mathrm{zu}$ vermitteln. Gute Rechtskenntnisse sind erforderlich, damit der Medizinstudierende seine Rechten und Pflichten schon im Anfangsstadium des Medizinstudiums kennt. So wird vermieden, dass möglicherweise schon in einer Famulatur oder später im PJ delegierte Aufgaben übernommen werden, die rechtlich nicht zulässig und mit einem erheblichen Haftungsrisiko belastet sind [14, 20].

\section{Fazit}

Rechtliche Aspekte sind inzwischen wesentlicher Bestandteil ärztlicher Tätigkeit. Demzufolge ist sowohl bei der Novellierung der ärztlichen Approbationsordnung als auch bei den Lernzielkatalo- gen dafür Sorge zu tragen, dass das Medizinrecht ausreichend und umfassend im Medizinstudium verankert wird. $\mathrm{Ob}$ die anstehende Reform durch den „Masterplan 2020" daran etwas ändern wird, bleibt abzuwarten [23]. Aktivitäten aus dem Frankfurter Institut für Rechtsmedizin tragen dazu bei, diesen Forderungen Rechnung zu tragen, indem die beiden Letztautoren im Gesetzgebungsverfahren (• Abb. 1) und bei der Curriculumsentwicklung bzw. -kartierung innerhalb des LOOOP-Netzwerks zur Weiterentwicklung der NKLM mitwirken. Gemäß der vorliegenden Umfrage entspricht eine umfangreichere rechtliche Lehre den Vorstellungen von über $90 \%$ der befragten Studierenden, die sich eine bessere medizinrechtliche Vorbereitung auf die spätere ärztliche Berufstätigkeit wünschen. Eine fächerübergreifende und umfassende Etablierung des Medizinrechts wurde von den beiden Sprechern der AG Arzt-, Medizin- und Patientenrecht (Parzeller, Verhoff) in Kooperation mit den weiteren Mitgliedern der AG (Chabiera, Duttge, Gaede und Wienke) und in der AG Professionelles Handeln Bereich Recht der Medizin in LOOOP eingearbeitet. Somit sollte eine Vermittlung von medizinrechtlichen Kenntnissen bereits ab dem ersten Studienjahr möglich sein. Aufgrund ihrer Nähe zum Recht kann die Rechtsmedizin dazu beitragen, die Vorbereitung auf das PJ sowie den Beruf als Ärztin oder Arzt deutlich zu verbessern und somit die Patientensicherheit zu erhöhen. Allerdingsist bei der Neugestaltung der Lehre im Medizinrecht zu berücksichtigen, dass für ein gutes Lehrangebot hinreichend zeitliche, finanzielle und personelle Mittel zur Verfügung gestellt werden müssen.

\section{Korrespondenzadresse}

\section{Dr. med. N. Kern}

Institut für Rechtsmedizin, Universitätsklinikum, Goethe-Universität Frankfurt Kennedyallee 104, 60596 Frankfurt am Main, Deutschland

n.kern@med.uni-frankfurt.de

Danksagung. Die Autoren bedanken sich bei Frau MSc Tina Koch für die Übersetzungshilfe des Abstracts. 
Funding. Open Access funding provided by Projekt DEAL.

\section{Einhaltung ethischer Richtlinien}

Interessenkonflikt. N. Kern und F. Holz geben an, dass kein Interessenkonflikt besteht. M.A. Verhoff und M. Parzeller geben an, aktiv bei der Reform des Medizinstudiums im LOOOP-Netzwerk, u. a. als AGSprecher mitzuwirken.

Für diesen Beitrag wurden von den Autoren keine Studien an Menschen oder Tieren durchgeführt. Für die aufgeführten Studien gelten die jeweils dort angegebenen ethischen Richtlinien.

Open Access. Dieser Artikel wird unter der Creative Commons Namensnennung 4.0 International Lizenz veröffentlicht, welche die Nutzung, Vervielfältigung, Bearbeitung, Verbreitung und Wiedergabe in jeglichem Medium und Format erlaubt, sofern Sie den/die ursprünglichen Autor(en) und die Quelle ordnungsgemäß nennen, einen Link zur Creative Commons Lizenz beifügen und angeben, ob Änderungen vorgenommen wurden.

Die in diesem Artikel enthaltenen Bilder und sonstiges Drittmaterial unterliegen ebenfalls der genannten Creative Commons Lizenz, sofern sich aus der Abbildungslegende nichts anderes ergibt. Sofern das betreffende Material nicht unter der genannten Creative Commons Lizenz steht und die betreffende Handlung nicht nach gesetzlichen Vorschriften erlaubt ist, ist für die oben aufgeführten Weiterverwendungen des Materials die Einwilligung des jeweiligen Rechteinhabers einzuholen.

Weitere Details zur Lizenz entnehmen Sie bitte der Lizenzinformation auf http://creativecommons.org/ licenses/by/4.0/deed.de.

\section{Literatur}

1. Approbationsordnung für Ärzte (ÄApprO 2002) vom 27.Juni 2002 (BGBI.IS. 2405), zuletzt geändert durch Artikel 3 des Gesetzes vom 16. März 2020 (BGBI. I S. 497); www.gesetze-im-internet.de/ appro_2002/ÄApprO_2002.pdf; zugegriffen: $1 \overline{1}$. Mai 2020

2. Bundesministerium für Gesundheit (2019) Arbeitsentwurf Approbationsordnung für Ärzte und Ärztinnen (ÄApprO). https:// www.bundesaerztekammer.de/fileadmin/user upload/downloads/pdf-Ordner/Stellungnahmen/ AEApprO_Arbeitsentwurf_SN-BAEK_Synopse_ final_24012020.pdf;.Zugegriffen:10. Mai 2020

3. Richter-Kuhlmann E (2016) Praktisches Jahr: Kein rechtsfreier Raum. Dtsch Arztebl 2016:10-12 (Medizin studieren)

4. LG Bielefeld (2014) Urt. v. 14.08.2013 - 18 JS 279/11 zur fahrlässigen Tötung nach intravenöser Injektion eines oralen Antibiotikums durch Medizinstudenten. Medizinrecht 32:579-581

5. Achterfeld C (2017) Rechtliche Rahmenbedingungen im Praktischen Jahr des Medizinstudiums. In: KatzenmeierC, Ratzel R(Hrsg) Festschrift für FranzJosef Dahm. Springer, Berlin, Heidelberg, New York, S1-16

6. Parzeller M, Amendt J, Zehner R et al (2017) Interdisziplinäre Lehre und Fortbildung - eine
Domäne der Rechtsmedizin. Arch Kriminol 239:117-128

7. Nold S, Anders S, Bajanowski T et al (2020) Inhaltliche und strukturelle Änderungen der rechtsmedizinischen Lehre in Deutschland. Rechtsmedizin 30:225-232

8. Anders S, Schwenn A, Püschel K (2007) Studentische Ausbildung im Fach Rechtsmedizin in Deutschland. Rechtsmedizin 17:153-158

9. Ondruschka B, Henze M, Schwarz M et al (2017) Wahltertial Rechtsmedizin im Praktischen Jahr. Rechtsmedizin 27:98-105

10. Anders S, Sperhake J, Andresen H, Weidtmann K, Püschel K (2005) Das Fach Rechtsmedizin und die neue Approbationsordnung für Ärzte. Rechtsmedizin 15:167-172

11. Ackel-Eisnach K, Raes P, Hönikl L et al (2015) Is German medical education research on the rise? An analysis of publications from the years 2004 to 2013. GMSZ Med Ausbild 32:Doc30

12. Woellert K (2019) Das Klinische Ethikkomitee: Ziele, Strukturen und Aufgaben Klinischer Ethik. Bundesgesundheitsblatt Gesundheitsforschung Gesundheitsschutz 62:738-743

13. Neuser M, Birngruber CG, Dettmeyer R (2019) Medizinrecht im Medizinstudium. Rechtsmedizin 29:477-483

14. Blank A, Dreßler J, Parzeller M (2015) „Ärztliche“ Aufklärung durch Studierende der Medizin. Rechtsmedizin 25:531-542

15. Galle P, Geldner G, Königsrainer A et al (2014) PJ-ler: Aufklärung in Sachen Aufklärungsgespräch. Lege artis $4: 141$

16. OLG Karlsruhe (2017) Urt. v. 29.01.2014 - 7 U 163/12 zur Selbstbestimmungsaufklärung durch einen Medizinstudenten im Praktischen Jahr. Medizinrecht 35:244-246

17. Katzenmeier C, Achterfeld C (2016) Delegation der Selbstbestimmungsaufklärung. Medizin Haftung - Versicherung, 1. Aufl. Springer, Berlin, Heidelberg, New York, S89-102

18. Wienke A, Sailer R (2014) Wirksame Aufklärung durch Medizinstudentin im Praktischen Jahr. Laryngorhinootologie 93:686-687

19. Rojahn J (2012) Ärztliche Tätigkeiten delegieren: Darauf sollten Sie achten! Lege artis 2:82-87

20. Parzeller M, Blank A, Zedler B et al (2017) Medizinstudium: Riskante Delegation von Aufgaben. Dtsch Arztebl 114(37):A1638-9

21. Bundesregierung, Entwurf eines Gesetzes zur Verbesserung der Rechte von Patientinnen und Patienten vom 15.8.2012, BT-Drs. 17/10488, S. 9

22. Franke KM (2010) Medizinrecht: Was dürfen Famulanten und PJler? - Packungsbeilage PJ. Via Medici 15:12-13

23. Rieger HJ (1991) Delegation ärztlicher Leistungen an Famuli. Dtsch Med Wochenschr 116:713-715

24. Wissenschaftsrat (2018) Empfehlungen der Expertenkommission zum Masterplan Medizinstudium 2020. https://www. wissenschaftsrat.de/download/archiv/7271-18. pdf;jsessionid=D16BFF2A630612D4CB3FEC5B2 D8C5FE7.delivery2-master? blob=publication File\&v=1.Zugegriffen: 10. Mai 2020(S.76, $135 \mathrm{ff}$ )

25. Taupitz J (2004) Rechtsmedizin und Medizinrecht? heute und morgen. Rechtsmedizin 14:163-168

26. Kiessling C, Rotthoff T, Schnabel KP et al (2019) 20 years of model curricula in German-speaking countries. GMS J Med Educ 36:Doc65

27. Chenot JF (2009) Undergraduate medical education in Germany. Ger Med Sci 7:Doc2

28. Heide S, Lessig R, Diers V et al (2016) Etablierung der Station "Leichenschau" in "SkillsLab" und E-Learning. Rechtsmedizin 26:90-96
29. FenwickA (2014) Medical ethics and law: assessing the core curriculum. J Med Ethics 40:719-720

30. Olick RS (2001) It's ethical, but is it legal? Teaching ethics and law in the medical school curriculum. Anat Rec 265:5-9

31. Preston-Shoot M, McKimm J (2010) Prepared for practice? Law teaching and assessment in UK medical schools. J Med Ethics 36:694-699

32. Koehler N, McMenamin C (2012) How relevant is undergraduate medical law teaching to clinical practice? A graduates' perspective. J Law Med 20:380-390

33. Parker M, Willmott L, White B et al (2018) Law as clinical evidence: a new constitutive model of medical education and decision-making. J Bioeth Inq 15:101-109

34. WHO Patient Safety Curriculum guide for medical schools. http://whqlibdoc.who.int/publications/ 2009/9789241598316_eng.pdf;. Zugegriffen: 10. Mai 2020

35. LOOOP-Projekt (2020) Nationaler Kompetenzbasierter Lernzielkatalog Medizin/ Kompetenzorientierte Gegenstandkataloge, Gemeinsame Entwicklungsplattform. https://gk. impp.de/zend/.Zugegriffen: 11. Mai 2020

36. IMPP (2019) Gegenstandskatalog für den Zweiten Abschnitt der Ärztlichen Prüfung, 5. Aufl., S88-89 